\title{
Estimating Cardiac Output from Arterial Blood Pressure Waveforms: a Critical Evaluation using the MIMIC II Database
}

\author{
JX Sun, AT Reisner, M Saeed, RG Mark \\ Harvard-MIT Division of Health Sciences and Technology, MIT, Cambridge, MA, USA
}

\begin{abstract}
Cardiac output (CO) estimation using arterial blood pressure $(A B P)$ waveforms has been an active area of physiology research over the past century. However, the effectiveness of the estimators has not been extensively studied in a clinical setting. In this paper, we evaluate 11 wellknown CO estimators using clinical radial ABP waveforms from the Multi-Parameter Intelligent Monitoring for Intensive Care II (MIMIC II) database, using thermodilution CO (TCO) as reference for comparison. We compare estimations to 988 TCO measurements in 84 patients, totaling 165 hours of ABP waveforms sampled at $125 \mathrm{~Hz}$. As a necessary step for producing absolute $\mathrm{CO}$ estimates, we also present 3 methods of calibrating the estimators, each tailored towards a different use model. The results show that the standard deviation of error between TCO and the best $\mathrm{CO}$ estimators is approximately $1 \mathrm{~L} / \mathrm{min}$ for absolute $\mathrm{CO}$ estimates. For relative estimates without calibration, the best CO estimator has $18 \%$ error at 1 standard deviation.
\end{abstract}

\section{Introduction}

Cardiac output (CO) is a key parameter in assessing circulatory function. The nominal $\mathrm{CO}$ value for a healthy human is about 5 liters per minute. Currently in clinical practice, the gold standard for $\mathrm{CO}$ measurement is thermodilution CO (TCO), which involves the insertion of a Swan-Ganz catheter into the pulmonary artery. Administered primarily in intensive care units (ICUs), TCO is usually measured intermittently, is very invasive, and may cause severe complications. It would be a tremendous asset to healthcare if one could determine CO accurately, reliably, and continuously using less invasive, indirect methods. Indeed, in the past century, over a dozen schemes have been proposed and developed to estimate $\mathrm{CO}$ using arterial blood pressure (ABP) waveforms obtained from a patient far less invasively. Some of these estimators rely on elaborate models of the heart and vasculature while others use artificial intelligence methods such as pattern matching and classification trees. The published estimators have not been extensively evaluated with a large set of clinical $\mathrm{ABP}$ waveforms, hence the performance of $\mathrm{CO}$ estimation is still uncertain. Studies in the past have mostly been conducted on a small set of subjects under well-controlled laboratory conditions. It is entirely possible that there will be circumstances in real world clinical practice in which these indirect methods produce inaccurate estimates.

The Multi-Parameter Intelligent Monitoring for Intensive Care II (MIMIC II) database has physiologic waveform data from over 3500 ICU patients hospitalized at Beth Israel Deaconess Medical Center, Boston, USA. The database has about 100 patient records that contain ABP waveforms and TCO measurements simultaneously. Our goal is to evaluate 11 of the $\mathrm{CO}$ estimators on a suitable subset of these patients using TCO as reference standard.

\begin{tabular}{lr}
\multicolumn{2}{c}{ Table 1. CO Estimators } \\
\hline Estimator & CO $=k \cdot$ below \\
\hline Mean Pressure & $P_{\text {mean }}$ \\
Windkessel [1] & $P_{\text {pulse }} \cdot \mathrm{HR}$ \\
Systolic Area [2] & $A_{\text {sys }} \cdot \mathrm{HR}$ \\
Warner Time Correction [3] & $\left(1+\frac{T_{\text {sys }}}{T_{\text {dias }}}\right) A_{\text {sys }} \cdot \mathrm{HR}$ \\
Liljestrand \& Zander [4] & $\left(\frac{P_{\text {pulse }}}{P_{\text {sys }}+P_{\text {dias }}}\right) \cdot \mathrm{HR}$ \\
Herd [5] & $\left(P_{\text {mean }}-P_{\text {dias }}\right) \cdot \mathrm{HR}$ \\
Corrected Impedance [6] & Variant of systolic area \\
Nonlinear compliance [7] & complex formula \\
RMS-Simplified form of [8] & $\sqrt{\left\langle\left(P(t)-P_{\text {mean }}\right)^{2}\right\rangle} \cdot \mathrm{HR}$ \\
Exponential best fit [9] & fit curve to diastolic decay \\
3-element model-variant of [10] & nonlinear, time-varying model \\
\hline
\end{tabular}

RMS, root-mean-square. HR, heart rate. $A_{s y s}$, area under systolic region of ABP. $T_{\text {sys }}, T_{\text {dias }}$ durations of systole, diastole.

\section{Overview of CO Estimators}

Table 1 lists the $11 \mathrm{CO}$ estimators analyzed in this study. All expressions given in the table are proportional to $\mathrm{CO}$. The proportionality factor encapsulates factors such as arterial compliance and peripheral resistance that are not usually obtainable from $\mathrm{ABP}$ waveforms.

The relationship between $\mathrm{ABP}$ and $\mathrm{CO}$ can be under- 
stood via the lumped-parameter Windkessel RC circuit model (Figure 1) of the cardiovascular system. In the model, electrical current is analogous to blood flow $Q(t)$ and electrical voltage to blood pressure $P(t)$. The current source models the heart as a pulsatile pump. The parallel resistor-capacitor combination models peripheral resistance and arterial compliance. In the simplest form, the current source generates a periodic impulse train, which gives the ABP waveform $P(t)$. From circuit theory, it can be shown that in steady state, the change in amplitude of the $\mathrm{ABP}$ waveform is proportional to the amount of blood ejected by the heart from each impulse. Thus, the Windkessel CO estimator suggests that pulse pressure is proportional to stroke volume, which yields $\mathrm{CO}$ when multiplied by heart rate. A few estimators are based on the Windkessel model, but use other features of the waveform such as the exponential decay of the ABP during diastole.

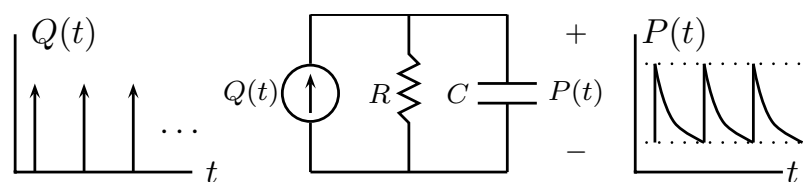

Figure 1. The Windkessel RC Circuit Model.

There are several major simplifying assumptions in the model that cause discrepancies from the real cardiovascular system. First, the entire venous circulation is neglected. Second, the real heart does not eject blood instantaneously. Finally, not only do arterial resistance and compliance vary over a cardiac cycle, but also it is more accurate to model the vasculature using a distributed system rather than lumped parameters. Therefore, in an attempt to produce more accurate results, some estimators have been developed using more complex models such as nonlinear arterial compliances and resistances, as well as distributed models in which the blood vessels are no longer viewed as lumped RC elements.

\section{Methods}

\subsection{Implementation}

Figure 2 presents the high-level data flow diagram of our system. The first block interfaces with the MIMIC II database to extract 10-minute ABP waveform segments centered at each TCO measurement.

The next 3 blocks use signal processing techniques to extract clean features of the ABP waveform required by each $\mathrm{CO}$ estimator such as systolic pressure, diastolic pressure, mean pressure (MAP), heart rate, etc. Since most features can be obtained from each beat in the ABP waveform, an onset detection algorithm [11] is used for beat isolation. The features are then median filtered to obtain aver-

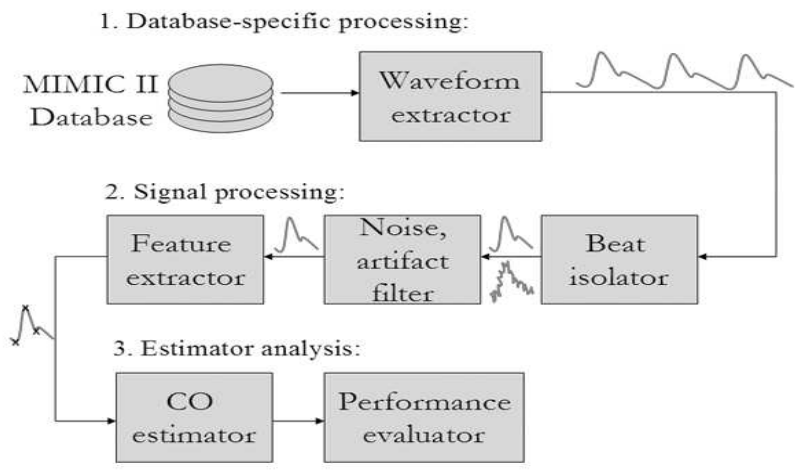

Figure 2. Data flow diagram for $\mathrm{CO}$ estimation.

age statistics for each 1-minute waveform segment. Since MIMIC II data is collected in a far less controlled environment than a typical research experiment setting, waveforms are prone to noise and artifact corruption. To address this problem, an algorithm that identifies bad waveform segments was designed. For each beat, the filter outputs a binary signal quality index (SQI), with ' 0 ' indicating clean and ' 1 ' indicating corrupted. Figure 3 shows an example of an ABP waveform marked with several features.

The third component implements each $\mathrm{CO}$ estimator, using extracted features of the $\mathrm{ABP}$ waveform as input. Since each estimator produces values proportional to the estimated CO, at least one calibration with TCO is necessary to obtain an absolute $\mathrm{CO}$ estimate in $\mathrm{L} / \mathrm{min}$.

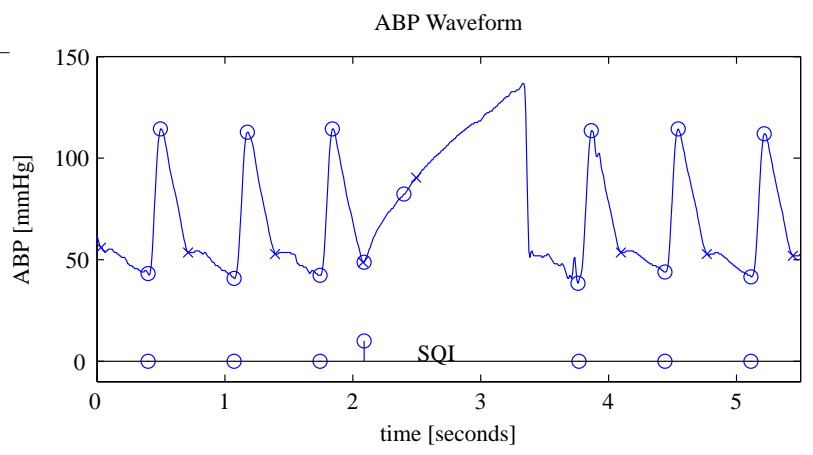

Figure 3. Radial ABP waveform. $P_{\text {sys }}, P_{\text {dias }}$ are marked on waveform as circles. End-of-systole detections are marked as crosses. SQI is shown as a stem plot on the bottom, flagging any corrupted beat at the onset time.

\subsection{Calibration and Evaluation}

We calibrate each estimator in three ways (denoted as C1, C2, C3), each tailored towards a different use model. In two of the calibration methods, we use a vector-based approach. For a patient with $N$ TCO measurements, we construct an $N$-dimensional column vector, with one di- 
mension for each measurement:

$$
\begin{array}{llll}
\text { Reference CO (TCO): } & \boldsymbol{r}=\left[\begin{array}{llll}
r_{1} & r_{2} & \cdots & r_{N}
\end{array}\right]^{\mathrm{T}} \\
\text { Uncalibrated estimate: } & \boldsymbol{x}=\left[\begin{array}{llll}
x_{1} & x_{2} & \cdots & x_{N}
\end{array}\right]^{\mathrm{T}} \\
\text { Calibrated estimate: } & \boldsymbol{q}=k \boldsymbol{x} & &
\end{array}
$$

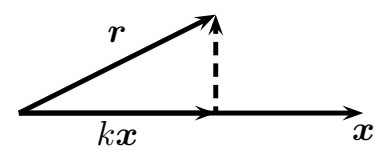

Figure 4. Visualizing TCO and estimated CO data as vectors. For $\mathrm{C} 1$, we choose $k$ to minimize error (magnitude of the dashed vector).

As shown in Figure 4, the magnitude of error normalized over TCO becomes

$$
\text { error }=\frac{\|\boldsymbol{r}-\boldsymbol{q}\|}{\|\boldsymbol{r}\|}
$$

For optimal calibration (C1), we choose a single constant $k$ to minimize the mean square error. Using linear algebra, the optimal $k$ is given as:

$$
k=\frac{\boldsymbol{r}^{\mathrm{T}} \boldsymbol{x}}{\boldsymbol{x}^{\mathrm{T}} \boldsymbol{x}}
$$

C1 calibration is useful in obtaining a lower bound of error for each estimator. However, C1 would be unsuitable in a live clinical setting, in which we do not know future values of TCO and ABP waveforms. Therefore, for online estimation, we update our optimal $k$ using previous data points. For the $i$-th $k$, we calibrate using the previous $(i-1)$-dimensional vector:

$$
k_{i}=\frac{\boldsymbol{r}_{i-1}^{\mathrm{T}} \boldsymbol{x}_{i-1}}{\boldsymbol{x}_{i-1}^{\mathrm{T}} \boldsymbol{x}_{i-1}}
$$

Now the calibrated estimate becomes:

$$
\boldsymbol{q}=\left[\begin{array}{llll}
k_{1} x_{1} & k_{2} x_{2} & \cdots & k_{N} x_{N}
\end{array}\right]^{\mathrm{T}}
$$

Often times, TCO is administered sparingly in ICUs. Therefore, it is also useful to know the estimator performance by calibrating only to the first TCO measurement:

$$
k=\frac{r_{1}}{x_{1}}
$$

\subsection{Relative CO estimation}

Outside of the ICU setting, TCO is likely unavailable; thus, we cannot calibrate to produce an absolute $\mathrm{CO}$ estimate. However, it is still useful to know relative fractional changes in CO. For example, if an uncalibrated estimator output decreased from 4000 to 2000 , we would like to know if the true $\mathrm{CO}$ has decreased by a similar fractional amount. For evaluation, we define percentage changes in TCO and each uncalibrated CO estimator as:

$$
\boldsymbol{X}=\frac{\boldsymbol{x}-\bar{x}}{\bar{x}} \quad \boldsymbol{R}=\frac{\boldsymbol{r}-\bar{r}}{\bar{r}}
$$

$\bar{r}$ and $\bar{x}$ are averages in TCO and CO estimator output, respectively. To report error, we examine the difference between $X$ and $R$. For example, if $X=0.3$ and $R=0.4$, the magnitude of error would be reported as 0.1 , or $10 \%$.

\subsection{Patient Selection}

We want to evaluate the $\mathrm{CO}$ estimators on patients that have relatively clean ABP waveforms and a significant number of TCO measurements. We accept patients in the MIMIC II database if all of the following are true:

1. SQI flags $\leq 20 \%$ of beats in ABP waveform.

2. Patients with $\geq 5$ TCO measurements.

3. Patients that do not have intra-aortic balloon pumps or with abnormal aortic or tricuspid valve function.

Based on these criteria, 84 patients were identified, each averaging 12 TCO measurements. Some statistics for the population is listed in Table 2.

Table 2. Subject Population Statistics

\begin{tabular}{lcc}
\hline Parameter & Mean & Range \\
\hline Age [years] & 70 & $40-95$ \\
TCO [L/min] & 5.3 & $2-12$ \\
$\Delta$ TCO per patient [L/min] & 2.5 & $1.5-6$ \\
\hline
\end{tabular}

\section{Results and Discussion}

The first 3 columns of Table 3 list the standard deviation of error in liters per minute between TCO and each estimator for the 3 different calibrations for absolute $\mathrm{CO}$ estimates. The last column of the table lists the percentage error at 1 standard deviation for relative $\mathrm{CO}$ estimates. The 95\% confidence intervals are about twice the values in the table. Figure 5 shows a Bland-Altman plot for the Liljestrand \& Zander estimator. The standard deviation and $95 \%$ confidence intervals are marked by a pair of solid lines and a pair of dashed lines, respectively.

From these results, we see that the best $\mathrm{CO}$ estimation methods give errors of approximately $1 \mathrm{~L} / \mathrm{min}$. For C1calibration, the lowest error is $0.92 \mathrm{~L} / \mathrm{min}$. Although this error may seem large, studies [12] have shown that TCO itself has errors of $10 \%$ at the $1 \mathrm{std}$ dev bound. Therefore, if a TCO measurement reads $5 \mathrm{~L} / \mathrm{min}$, there may exist an error up to $0.5 \mathrm{~L} / \mathrm{min}$. It is interesting to note that the mean pressure and Windkessel estimators, the most primitive estimators of $\mathrm{CO}$, have less error than some of the more advanced ones. Because some of the error distributions were 
non-Gaussian, we employed the Kolmogorov-Smirnov test to see if the top five estimators were statistically significant better predictors of $\mathrm{CO}$ than mean pressure (these best five were identified in a previous, smaller pilot study). The threshold for significance was $p<0.01$, after a Bonferroni correction for multiple $(n=5)$ comparisons. The Liljestrand \& Zander $\left(p=6.67 \times 10^{-6}\right)$ and Corrected impedance ( $p=4 \times 10^{-4}$ ) estimators were significantly superior to mean pressure, based on their error distributions resulting from the $\mathrm{C} 1$ calibration method.

Table 3. Estimation error in $\mathrm{L} / \mathrm{min}$ at 1 standard deviation with 3 different calibration methods, sorted from best to worst. The last column lists the percentage error for relative estimation without calibration.

\begin{tabular}{lllll}
\hline Error at $1 \sigma$ & C1 & C2 & C3 & rel \\
\hline Liljestrand \& Zander & 0.92 & 1.01 & 1.23 & $18 \%$ \\
Systolic area & 0.96 & 1.06 & 1.29 & 19 \\
Corrected impedance & 1.00 & 1.09 & 1.29 & 20 \\
Time correction & 1.02 & 1.12 & 1.35 & 20 \\
Mean pressure & 1.02 & 1.11 & 1.43 & 20 \\
Windkessel & 1.04 & 1.16 & 1.37 & 20 \\
Root-mean-square & 1.04 & 1.18 & 1.38 & 21 \\
Herd & 1.24 & 1.37 & 1.55 & 25 \\
Nonlinear compliance & 1.28 & 1.46 & 1.72 & 26 \\
3-element model & 1.45 & 1.68 & 2.51 & 30 \\
Exponential best fit & 1.80 & 3.29 & 4.54 & 37 \\
\hline
\end{tabular}

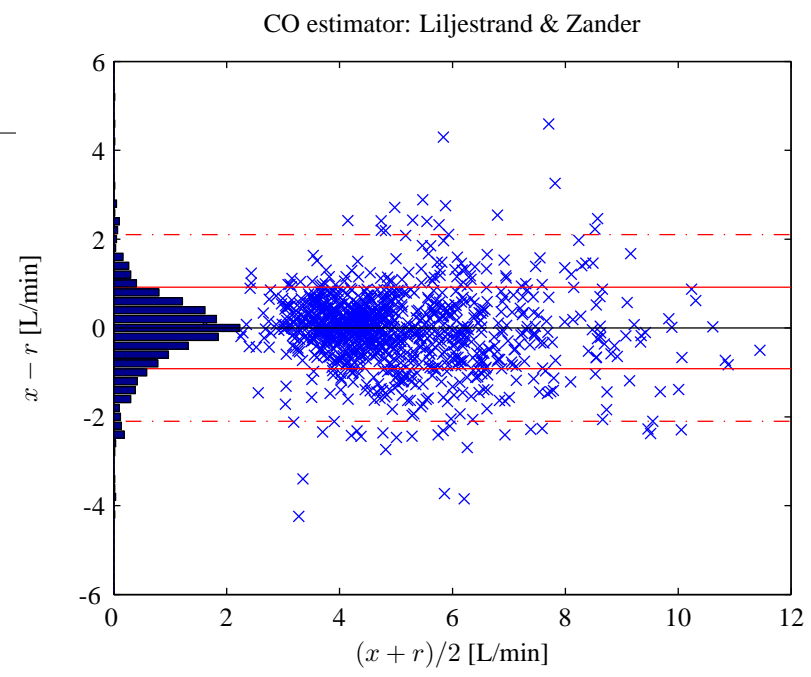

Figure 5. Bland-Altman plot for comparing the Liljestrand and Zander estimator $(x)$ to TCO $(r)$ with $\mathrm{C} 1$ optimal calibration. The error histogram is shown on the left. The solid lines show 1 std dev bounds, and the dashed lines show $95 \%$ confidence intervals.

\section{Acknowledgments}

This research was supported by grant R01 EB001659 from the National Institute of Biomedical Imaging and Bioengineering and by the Center for the Integration of Medicine and Innovative Technology (CIMIT).

\section{References}

[1] Erlanger J, Hooker B. Johns Hopkins Hospital Report 1904; 12:147-378.

[2] Wesseling K, Nicols W, Wit B, H. W. A beat-to-beat cardiac output computer. In Proceedings of the third International Conference on Medical Physics. 1972; .

[3] Warner H, Swan H, Connolly D, Tompkins R, Wood E. Quantitation of beat-to-beat changes in stroke volume from the aortic pulse contour in man. Journal of Applied Physiology 1953;5:495.

[4] Liljestrand G, Zander E. Experimental Medicine 1940; 59:105.

[5] Herd J, Leclair N, Simon W. Arterial pressure pulse contours during hemorrhage in anesthetized dogs. Journal of Applied Physiology 1966;21.

[6] Wesseling K, Wit B, Weber J, Smith N. A simple device for the continuous measurement of cardiac output. Advanced Cardiovascular Physiology 1983;5.

[7] Godje O, Lamm P, Schmitz C, Theil M, B. R. Continuous, less invasive, hemodynamic monitoring in intensive care after cardiac surgery. Thoracic Cardiovascular Surgery 1998; 46:242-249.

[8] Jonas M, Hett D, Morgan J. Real time, continuous monitoring of cardiac output and oxygen delivery. International Journal of Intensive Care 2002;9.

[9] Bourgeois J, Gilbert B, Donald D, Wood E. Characteristics of aortic diastolic pressure decay with application to continuous monitoring of changes in peripheral vascular resistance. Circulation Research 1974;35:56-66.

[10] Wesseling K, Jansen R, Settels J, Schreuder J. Computations of aortic flow from pressure in humans using a nonlinear, three-element model. Journal of Applied Physiology $1993 ; 74$.

[11] Zong W, Heldt T, Moody G, Mark R. An open-source algorithm to detect onset of arterial blood pressure pulses. In Computers in Cardiology 2003. Los Alamitos: IEEE Computer Society Press, 2003; 259-262.

[12] Stetz C, Miller R, Kelly G, Raffin T. Reliability of the thermodilution method in the determination of cardiac output in clinical practice. Am Rev Respir Dis 1982;126.

Address for correspondence:

James X. Sun

MIT, Room E25-505, 77 Mass Ave, Cambridge, MA 02139, USA

xinsun@mit.edu 